Association for Information Systems AIS Electronic Library (AISeL)

ECIS 2000 Proceedings

European Conference on Information Systems

2000

\title{
The Electronic Patient Record as an Organizational Artifact
}

Pieter Toussaint

Technical University Delft, pietert@sepa.tudelft.nl

Marc Berg

Erasmus University of Rotterdam, m.berg@hmg.eur.nl

Follow this and additional works at: http://aisel.aisnet.org/ecis2000

\section{Recommended Citation}

Toussaint, Pieter and Berg, Marc, "The Electronic Patient Record as an Organizational Artifact" (2000). ECIS 2000 Proceedings. 135. http://aisel.aisnet.org/ecis2000/135

This material is brought to you by the European Conference on Information Systems (ECIS) at AIS Electronic Library (AISeL). It has been accepted for inclusion in ECIS 2000 Proceedings by an authorized administrator of AIS Electronic Library (AISeL). For more information, please contact elibrary@aisnet.org. 


\title{
The Electronic Patient Record as an Organisational Artefact
}

\author{
Pieter Toussaint \\ Faculty of Technology, Policy and Management \\ Technical University Delft \\ The Netherlands \\ $\underline{\text { Pietert@sepa.tudelft.nl }}$ \\ Marc Berg \\ Institute of Health Policy and Management \\ Erasmus University Rotterdam \\ The Netherlands \\ M.Berg@bmg.eur.nl
}

\begin{abstract}
The research effort on Electronic Patient Records (EPR's) has rapidly increased in the last decade. Much of this research focussed on standardisation and technical realizations. We will describe such a research effort in this paper, and evaluate its success. Our main finding is that the lack of success of this specific research effort is mainly due to its technological bias. Although standards (both conceptual and technical) are important prerequisites for the realisation of an EPR, organisational issues are decisive for success. The role played by these organisational issues will be illustrated by analysing the findings of the case study presented in the paper. We will argue that research on EPR's should be more focussed on the role of an EPR as an organisational artefact that co-ordinates the work of health care professionals, in order to lead to successfull implementations.
\end{abstract}

\section{INTRODUCTION}

Health care changes. In [1] the authors discuss both internal and external reasons for this change. External stakeholders, such as insurance companies and the government, want more influence on the process of providing care to patients. Their objective is to increase the efficiency and effectiveness of the care process. Internal reasons for the change of health care are the further specialisation of the medical profession, and the more frequent occurrence of chronic diseases due to the increase of average age. These internal reasons imply a more intensive co-operation between health care professionals in the process of delivering care. This co-operation requires co-ordination of activities in time and place.

The Electronic Patient Record can play a major role in enabling and shaping these changes. In [2] two main functions that an EPR can play are discussed: accumulating and co-ordinating. The EPR accumulates information on patients. That is, it stores and aggregates patient data, and as such provides an overview of the care history related to the patient. It must be stressed that in doing this the EPR is an active artefact, because it constructs a view (or multiple views) of the patient record in the process of accumulating. The EPR also co-ordinates the work of different health care professionals. It enables the sharing of knowledge on a patient, and can passively (by making knowledge accessible for all health care professionals) and actively (by notifying or alerting professionals) influence their work processes.

This co-ordination function can result in a change in the cooperation between health care professionals. Because of this effect the EPR is often claimed to enable the emergence of 'virtual' health care teams. This claim is evaluated in this paper against the results of implementing an EPR in order to support shared care for diabetes patients, which was done as a pilot of a European R\&D project on EPR's called Synapses [3].

The Synapses project ran from 1995 until the end of 1998. It involved 26 partners from 14 different European countries. The budget for Synapses was 5.2 Million ECU. The project set out to solve problems of 
sharing medical record data between autonomous information systems, by providing generic and open means to combine health care records or dossiers consistently, simply, comprehensibly and securely, whether the data passes within a single health care institution or between institutions. The Synapses project developed the specifications of a server, acting as a mediator between information systems keeping parts of medical records (the so called feeder systems), and client applications, used for viewing medical records.

Currently, the electronic and paper records used by health care professionals are mostly held in islands of information. Therefore, sharing information across systems is very difficult and time consuming. Often, non-automated and non-efficient means of communication are used for sharing information. This is hampering the progress towards shared care and cost-containment.

These problems are clearly illustrated in the case of diabetic patients. The treatment of diabetic patients involves the general practitioner (GP), a nurse specialised in the treatment of diabetic patients and several health care professionals at the outpatient clinic, and various departments within the hospital. At the different stages of this process several flows of information occur between the health care professionals involved. In the Academic Medical Centre it was felt that the the efficiency and the effectiveness of communication between the health care professionals involved in this process was far from optimal, and therefore a re-engineering of the shared care process for diabetic patients was considered within the scope of a Synapses pilot. A major objective of the Academic Medical Centre was to adhere to the so-called St. Vincent declaration, a document compiled in 1989 with diabetes organisations, healthcare professionals and people with diabetes. Based on the objectives formulated in this declaration, and some local objectives, a number of goals were formulated for reorganising the diabetes care process: [4]

1. Increasing the self-regulation by the patient.

2. Increasing involvement of the treatment by first line healthcare professionals, such as general practitioners. In particular, patients suffering from diabetes mellitus type II, must be treated and monitored as much as possible by their general practitioner.

3. Diminishing the complications of diabetes mellitus. Some of the specific objectives are: 50 $\%$ less amputations, 33\% less blindness, and 33\% less kidney insufficiency caused by diabetes.
In order to achieve these objectives, intensive co-ordination of the treatment of diabetic patients is required. The goal of diabetes management is to keep blood glucose levels as close as possible to the normal (non-diabetic) range. The patient is responsible for day-to-day care. In addition, an endocrinologist monitors their physical condition and checks for complications. Also, people suffering from diabetes often see other specialists, such as: the ophthalmologist for eye examinations, podiatrists for foot care, dieticians for meal planning guidance, and diabetes educators for instructions on day-to-day care.

In principle diagnosis, treatment and monitoring patients suffering from diabetes mellitus type II, can be performed by general practitioners. It requires no knowledge to the degree of a specialist. However, this is not common practice, because most general practitioners feel uncomfortable with their knowledge, skills and policy of treating diabetes patients.

The basic requirements that followed from these objectives is that information on the physical condition of a diabetes patient can be shared by all professionals involved in the care process, and that guidelines on treating these patients are exchanged between endocrinologist and general practitioners. A number of Dutch Synapses partners (software company HISCOM at which one of the authors was employed, Academic Medical Centre in Amsterdam, and two General Practitioners practices in Amsterdam) co-operated in the development of a record server that is compliant with the Synapses specifications, in order to enable the sharing of the medical entities included in the DiabCare dataset between these health care providers. The DiabCare dataset is a set of data elements, agreed upon at the European level, encompassing all information relevant for monitoring the stage of illness, the therapy and secondary complications in the case of diabetes patients [5]. Furthermore, the record server provides access to a database with guidelines or protocols for treating diabetes patients.

In $[6,7]$ the technical solution has been reported and discussed. In this paper we will focus on the evaluation of the use of the system. First, in section II, we briefly describe the Synapses solution using the five viewpoints advocated by the Open Distributed Processing (ODP) standard [8]. Then, in section III, we evaluate the system use, and discuss the findings.

\section{THE SYNAPSES SOLUTION}

The Synapses solution can be seen as the realisation of a distributed health care record system, constructed out of different heterogeneous component systems. The ODP standards initiative [8] defines five 
perspectives, called viewpoints, from which distributed systems can be described. These five viewpoints are:

- the enterprise viewpoint - is concerned with the business environment in which the system has to operate;

- the information viewpoint - is concerned with the information to be stored and processed by the system;

- the computational viewpoint - is concerned with a description of the system as a set of objects that interact at interfaces;

- the engineering viewpoint - is concerned with the mechanisms supporting system distribution;

- the technology viewpoint - is concerned with the detail of components from which the distributed system is constructed.

In this paper the Synapses server, as developed for supporting shared care for diabetes patients, is described from each of the five ODP viewpoints. We will start with a description from the enterprise viewpoint, and work from there all the way down to the technology viewpoint.

\section{A. THE ENTERPRISE VIEWPOINT}

The enterprise viewpoint describes the distributed system to be developed in the business environment in which it will operate. Main focus of such a description is the relations between the participants in the business process to be supported. Our model from this viewpoint will be illustrated by means of a small number of scenarios exemplifying four typical co-operative activities taking place within the realm of diabetes care.

1. The general practitioner consults the internist on the correct diagnosis.

2. The general practitioner consults the internist on the choice treatment to provide.

3. The general practitioner consults the internist on an issue related to conducting the treatment chosen.

4. The general practitioner refers the patient to the hospital (transfers the care to the internist and the diabetic nurse).

In the following figure these scenarios are depicted graphically. We have divided the care activity into five sub-activities: taking the anamnesis, performing some examinations, diagnosing, planning a treatment, and conducting a treatment. The arrows between the sub-activities are labelled with a number indicating to which scenario the arrow belongs. This figure does not advocate a strict ordering of phases, with clear transitions between them, as the model of medical care. It is only included to indicate the different stages of the care process at which communication and co-ordination between different health care providers takes place in the four scenarios.

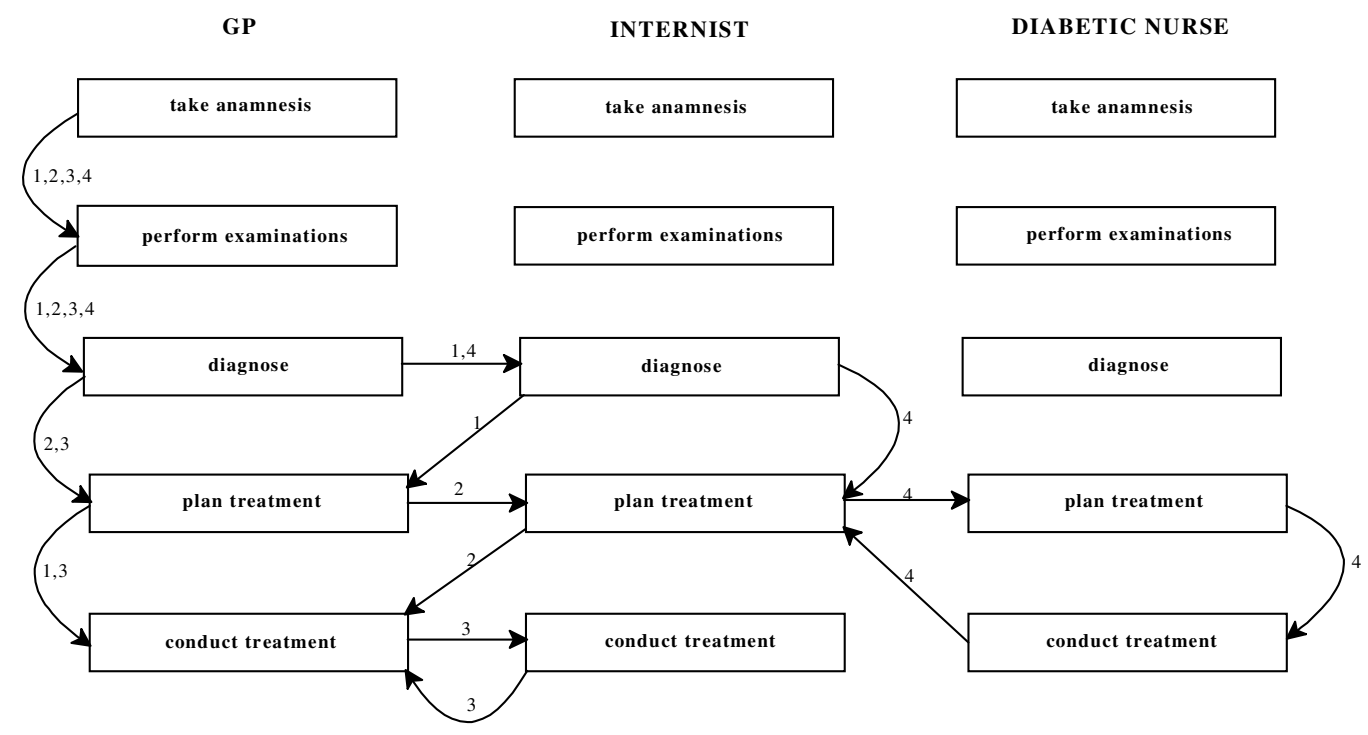

Fig. 1. Four scenarios that can be distinguished in the process of shared care for diabetes patients 
We have deliberately formulated these scenarios without reference to technology. Only human actors participate in the process of communicating and (as a result) co-ordinating behaviours. Our aim in developing the Synapses server was to enable communication and coordination by means of information and communication technology. So, the Synapses server would be an actor in itself, enabling, but also constraining, the interworking of the human actors in this specific network. This role of the Federated Health Care Record (FHCR) is in line with the ideas on the role of EPR's as expressed in [2].

$B$.

\section{THE INFORMATION VIEWPOINT}

Enabling the communication of information between human actors is the key role played by the FHCR. As noted before, this information comes in two types: information on the physical condition of diabetes patients, and information on guidelines or protocols for diabetes treatment. The last type was exchanged as free texts, and is therefore not detailed here. The first type of information was structured according to the prescriptions coming from two complementary standardisation results: a standard on Electronic Health Care Records [9], and a specification of the dataset required for diabetes treatment and monitoring [5]. Information coming from different sources was integrated into this structure, and presented to the different health care providers, giving them a uniform and integrated view the diabetes record of a patient.

In the figure below a simplified version of the model is described as an object model, using the UML notation [10]. A RecordFolder (record of one patient) consists of one or more episodes of care. Each episode of care consists of one Episode_of_Care_Description and zero or more Episode_of_Care_Entries. An episode of care entry specialises into an element of the diabcare dataset. Both description and entry inherit from the ENV 12265 class ComRIC, which represents the smallest meaningful, autonomous set of information that can be communicated.

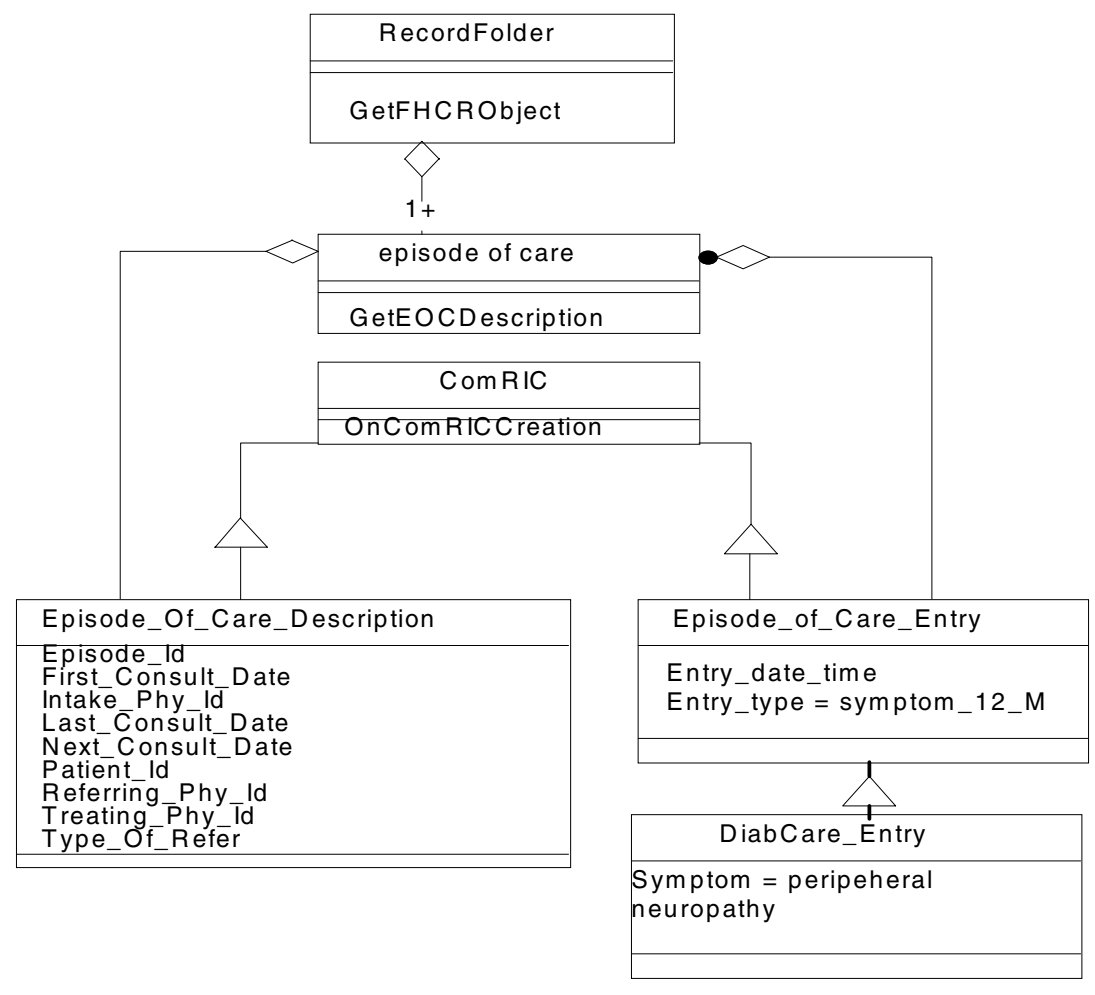

Fig. 2. The object model of the Synapses server 


\section{THE COMPUTATIONAL VIEWPOINT}

The integrated information model is distributed over the computational components making up the distributed system. We distinguish between three types of components: client applications, feeder systems and middleware components. The instances of these three types encountered in our pilot study are discussed below:

1. Client application: This component offers health care providers involved in the process of providing shared care for diabetic patients, a uniform view on the distributed patient record, as well as access to the repository containing the guidelines or protocols.

2. Feeder system: This component stores (parts of) the patient record.

3. Synapses Server: this middleware component is responsible for making the distributed character of the FHCR transparent to client applications. The view offered on the patient record must be compliant to the object model presented in the section on the information viewpoint.

4. Client Adapter: If non-synapses compliant client applications are used, information flows between the client application and the synapses server must be syntactically and semantically converted. This is the function of the client adapter.

5. Feeder Adapter: Currently a number of information systems are in use for registering parts of the medical record. In our pilot study diabetes related information is stored in systems used by the general practitioners and in a hospital information system. These systems are not Synapses compliant. Their data is structured differently, they use different keys for patient identification, different security rules and different services for accessing the data. These differences are to be dealt with by the feeder adapters. These adapters wrap the feeders in such a way that they become Synapses compliant.

The components have well-defined interfaces. We have described these interfaces using OMG IDL [11].

\section{THE ENGINEERING VIEWPOINT}

The engineering viewpoint further details the computational viewpoint. It specifies how the computational components are connected using a CORBA based integration solution. It is the last step in the specification before technologies and platforms can be selected for realising the distributed system.

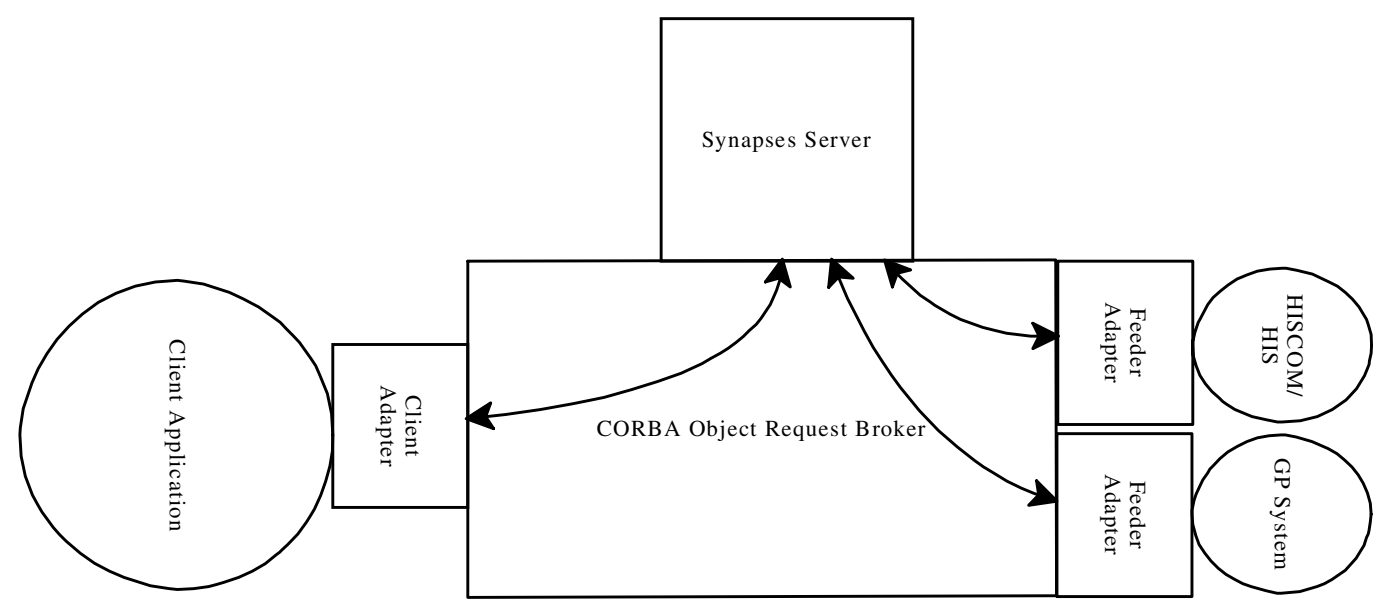

Fig. 3. the engineering architecture for the Synapses solution

With regard to the client application and the feeder system components distinguished in the computational viewpoint, we also indicate which specific instances of these components will be chosen for the pilot study. So, the engineering viewpoint marks the transition from the generic Synapses solution to the specific pilot study. We have two instances of the feeder system component: a GP system and the HISCOM/HIS. They are wrapped by means of an adapter in order to make them Synapses compliant. Requests from the client application are translated in the client adapter and passed via the 
Object Request Broker (ORB) to the synapses server. Here the request is interpreted and translated into requests for the feeder systems. These requests are passed to the appropriate feeders via the ORB. Responses follow the same route in the opposite direction.

\section{E. THE TECHNOLOGY VIEWPOINT}

The components distinguished in the engineering viewpoint are realised using several technologies and hardware platforms:

- Client application: Each of the health care providers involved in the process of shared care for diabetes patients, has his own client application for accessing the patient record. Two clients were used in the pilot:

- an application developed in Delphi, running on a PC under Windows95, already in use in the hospital

- an application developed in Java, used by the GP

- The Synapses Server: The server was developed in Visual $\mathrm{C}++$ and runs on a PC under windowsNT 4.0

- The adapters: The adapters were also developed in Visual $\mathrm{C}++$, and run on a PC under WindowsNT 4.0

- The ORB: For the CORBA Object Request Broker we have chosen ORBIX 2.0 from Iona ltd. This product offers an extensive implementation of the CORBA 2.0 specification. It uses a TCP/IP based network protocol for supporting communication.

- $\quad$ GP feeders: One of the GP feeder systems runs under Windows95 on a PC and the other GP feeder system runs under UNIX.

- HIS: The HISCOM HIS runs under UNIX.

\section{EVALUATION AND DISCUSSION}

In the second half of 1998 the system was implemented at the validation site, and its use was evaluated. In this section we will present and discuss the main findings. A more extensive report on this can be found in the final report on the Synapses project, available at [12].

As stated above, the main objective of the FHCR was that it would act as a co-ordinator of co-operation between different health care professionals involved in the shared care for diabetes patients. The most important functions to be offered were enabling the sharing of medical records between the health care professionals, and the sharing of guidelines or protocols on diabetes treatment. These functions were realised, along the lines discussed in the previous section. Two major design constraints were central to the Synapses approach:

- (Inter) national standards had to be used if possible. As can be seen in the brief description of the Synapses solution in the previous section, this led to choices for technological standards, such as CORBA, as well as conceptual standards, such as the European standard on record architectures and the Diabcare dataset.

- Legacy systems already in use by the cooperating actors had to be integrated into the FHCR as autonomous components. This led to the development of the adapters, which act as wrappers, turning legacy systems into CORBA/Synapses components. This loosely coupled strategy reflects the nature of the professional network in which the health care professionals co-operate.

So, the FHCR as developed in the Dutch pilot adhered to international standards on medical record structures and distributed technologies. Furthermore, it was structured as a loosely coupled system, a structure that reflects the nature of the professional network of health care providers co-operating in shared care activities. However, the system was not a success. It was not used in clinical routine, and even worse, the health care professionals involved did not conceive it as a promising solution for their problems. The main reasons for their dis-satisfaction were:

- Small number of patients involved makes record useless,

- The records that are available are not complete. Only diabetes-related sub-set is accessible.

- FHCR clients were not integrated with legacy system clients.

- FHCR is a passive co-ordinator. Active means of communication are not implemented.

At first glance these reasons seem to relate to contingent features of the implementation. However, in our opinion these features are not that contingent at all. We will discuss each of the features below, and motivate that these relate to common problems encountered in Electronic Patient Record implementations.

First of all, the number of patients on which medical record information could be shared was very limited. Only 10 patients were involved. This limitation surely affects everything we want to say about the effects of the system as a co-ordinator of care, but it also highlights some problems with the implementation of these systems, that are not 
incidental. The first reason for this limited set of patients has to do with the privacy regulations in the Netherlands. In fact, no medical information may be shared or exchanged between health care professionals without explicit consent of the patient. How to handle this constraint in the realm of these type of systems that are aimed at enabling the sharing of medical information? In the Synapses pilot we decided to protect ourselves from legal problems by using a small group of patients, that were explicitly asked if they agreed with the pilot. The second reason for the size of the patient group was the fact that the endocrinologist was in the middle of a migration process from paper-based record keeping to electronic record keeping. Only a limited number of his patients were already 'in' his record system and from these patients only a selected part of their history had been included. So, the view on patient records that could be constructed from the electronic record system used by the endocrinologist was by no means comparable to the view contained in his paper-based archive.

The second reason relates to the completeness of the records. In the pilot we choose only to include information that was included in the Diabcare dataset. Especially the general practitioners involved considered this to be a severe limitation. They encountered patients suffering from diabetes, that were often visiting the hospital for non-diabetes related problems. They wanted a complete update of the things that happened to these patients in the hospital, and not an update restricted to the diabetesrelated activities only. In relation to this, they considered the restriction to a specific patient group artificial. This touches upon the problem of views, or professional specific aggregations of patient data. Each professional often needs a different view on the patient record. A view that relates to his or her specific role in the care process. Two important questions to be addressed here are: how can such a view be constructed from the fragments stored in the different feeder systems, and which data must be registered by the different health care professionals in order that such a view is constructable. But why should health care professionals register data with the sole purpose of facilitating the work of others. How can 'return on investment' be defined in this context? In the context of the Synapses pilot discussed in this paper, it was clear that both the hospital and the hospital professionals involved were not inclined to change work processes or speed up record implementation efforts in order to increase the quality of communication with general practitioners.

The third reason for the limited usefulness of the FHCR developed was that client applications were not integrated with the client applications already in use by the general practitioners, the endocrinologist and the diabetic nurse. Again, this may be seen as a strong indicator for the limitations of the evaluation presented here, but also in this case this limitation is not a mere incident. Strong integration of the record systems of the different professionals by means of integration of their client applications jeopardises the autonomy of these record systems, and as an effect also the autonomy of the health care professionals. This contradicts the very nature of the professional network in which the health care providers are participating. Client applications are closely intertwined with the activities a health care professional performs, and therefore, changes to these clients will only be acceptable if the incentives to change a way of working are clear to the professional. So, we may conclude that this is in general a difficult thing to achieve.

The last reason is that no active communication functions were implemented in the system. So, the system did not actively submit reminders or requests to participating health care professionals. The coordination role was passive. Implementation of a more active role requires a more thorough understanding of the process of knowledge sharing between health care professionals, and is as such not systematically addressed in the literature on medical informatics. There are still a lot of open questions in this field of study, and as a result all implementations of electronic patients records will have to deal with this lack of essential knowledge.

So, we may conclude that the disappointing result of the FHCR implementation within the Dutch Synapses pilot, is not the result of not using standard technology or standardised concepts but the result of a lack of insight into important characteristics of the legal and organisational environment in which this systems was realised. The effects of the privacy legislation and the migration process towards an electronic record system taking place in the hospital, were only noticed later on in the pilot project, and still the question how to address these issues properly is an open one. The issues of completeness of the record and the integration of FHCR clients with local record system clients relate to the trade off between autonomy and interdependency between the health care professionals being part of a shared care network. And the last point on the active role of the FHCR in the co-ordination of activities, pushes this issue even a little further. Extended with this functionality, the FHCR becomes an active actor in the network, therewith changing its structure. Is this acceptable for the health care professionals involved, and will they trust the FHCR as such?

The work within Synapses presented here can be seen as a typical example of the work done within medical informatics in the last decade (see for 
example [13] for an overview of current research). In the context of electronic patient records there has been a lot of attention for technical and medical informational issues, but hardly any for the organisational issues around the patient record. In our view the electronic patient record is mainly an organisation artefact that accumulates patient data and helps in co-ordinating the work activities of health care professionals. So, in order to design and implement such an artefact properly, we must thoroughly understand the characteristics of the health care professional network and the role of the electronic patient record in such a network. This research objective has been taken up in a three-year research project funded by the Dutch government that will run from the end of 1999 to the end of 2003. Both authors will participate in this project. In close co-operation with a number of hospitals we will analyse health care processes and derive design guidelines for electronic patient records.

\section{REFERENCES}

[1] Berg, M. et al., De Nacht Schreef Rood: Informatisering van Zorgpraktijken, 1999. Rathenau Instituut, Den Haag.

[2] M. Berg, “Accumulating and Coordinating: Occasions for Information Technologies in Medical Work", Computer Supported Cooperative Work (8), Kluwer Academic Publsihers, 1999, pp. 373-401.
[3] J. Grimson, et al., "SYNAPSES - Federated Health Care Record Server", in: Medical Informatics Europe'96, J. Brender, J.P. Christensen, J.-R. Scherrer, P. McNair (Eds.), 1996, pp. 695-699.

[4] Synapses Consortium, Synapses ODP specification, to be published at IOS press.

[5] Information on this data set and the project Diabcare $Q$-net can be found on the world wide web at: http://www.diabcare.de

[6] P.J. Toussaint, M. Kalshoven, M. Ros, H. van der Kolk, en O. Weier, 'Supporting Shared Care for Diabetes Patients: the Synapses solution', in: [12], 1997, pp. 393-397.

[7] P.J. Toussaint, Integration of Information Systems: a study in requirements engineering, Ph.D.thesis Leiden University, 1998.

[8] ODP, ISO/IEC DIS 10746, Part 1-4.

[9] P. Hurlen, Electronic Healthcare Record Architecture, 1995, CEN/TC 251/WG 1 N95-38.

[10] G. Booch, J. Rumbaugh, I. Jacobson, The unified modeling language user guide, Addison Wesley, 1999.

[11] J. Siegel, CORBA Fundamentals and Programming, John Wiley \& Sons, Inc., 1996.

[12] http://www.cs.tcd.ie/synapses/public/

[13] D.R. Masys (Ed.), Proceedings of the 1997 annual fall symposium of the American Medical Informatics Association, 1997. 